\title{
Independent sets in graphs with given minimum degree
}

\author{
Hiu-Fai Law Colin McDiarmid
}

September 26, 2012

\begin{abstract}
We consider numbers and sizes of independent sets in graphs with minimum degree at least $d$, when the number $n$ of vertices is large. In particular we investigate which of these graphs yield the maximum numbers of independent sets of different sizes, and which yield the largest random independent sets. We establish a strengthened form of a conjecture of Galvin concerning the first of these topics.
\end{abstract}

Given a graph $G$, let $\mathcal{I}(G)$ be the set of independent sets and let $i(G)=$ $|\mathcal{I}(G)|$; and for $k \geq 0$ let $\mathcal{I}_{k}(G)$ be the set of independent sets of order $k$ and let $i_{k}(G)=\left|\mathcal{I}_{k}(G)\right|$. Thus $i(G)=\sum_{k>0} i_{k}(G)$.

There are many extremal results on $i(G)$ and $i_{k}(G)$, where $G$ ranges over a certain family of graphs, for example, trees or regular graphs (see [1]-[3], [5]-[7],[10]). Here we investigate graphs with a given lower bound on their vertex degrees. For $d \geq 0$, let $\mathcal{G}_{n}(d)$ be the set of graphs of order $n$ with minimum degree at least $d$. (Always $n, k$ and $d$ will be integers.) We are interested in which of these graphs yield the maximum numbers of independent sets of different sizes, and which yield the largest random independent sets. Let us discuss numbers first.

Recall that the independence number $\alpha(G)$ is the maximum size of an independent set. Clearly $\alpha(G) \leq n-d$ for each $G \in \mathcal{G}_{n}(d)$. Recently, Galvin [3] proved that, for $n$ suitably larger than $d, i(G)<i\left(K_{d, n-d}\right)$ for any $G \in \mathcal{G}_{n}(d)$ that is not (isomorphic to) $K_{d, n-d}$. Moreover, he conjectured essentially that for any $d \geq 1$, there exist integers $N(d)$ and $C(d)$ such that for each $n \geq N(d), K_{d, n-d}$ maximizes $i_{k}$ over all graphs in $\mathcal{G}_{n}(d)$ for each $k$ satisfying $C(d) \leq k \leq n-d$; and he proved such a result in the case when $d=1$.

We shall see that this conjecture holds even if $d$ is allowed to grow slowly, and further we can take $C(d)=3$. Observe that we need $C(d) \geq 3$. For, 
each $n$-vertex graph has $i_{0}(G)=1$ and $i_{1}(G)=n$. Also $i_{2}(G)=\left(\begin{array}{l}n \\ 2\end{array}\right)-e(G)$, where $e(G)$ is the number of edges, and graphs $G \in \mathcal{G}_{n}(d)$ can have $i_{2}(G)>$ $i_{2}\left(K_{d, n-d}\right)$. (For example, if $d$ is fixed and $n$ is large and even, $K_{d, n-d}$ has $d(n-d) \sim d n$ edges, whereas a $d$-regular graph has $d n / 2$ edges.) We shall show:

Theorem 1. Let $1 \leq d=d(n)=o\left(n^{1 / 3}\right)$. Then for all sufficiently large $n$, for each graph $G \in \mathcal{G}_{n}(d)$ and each $k \geq 3$ we have $i_{k}(G) \leq i_{k}\left(K_{d, n-d}\right)$; and if $G$ is not $K_{d, n-d}$ then $i_{2}(G)+i_{4}(G)<i_{2}\left(K_{d, n-d}\right)+i_{4}\left(K_{d, n-d}\right)$, and so $i(G)<i\left(K_{d, n-d}\right)$.

A graph $G \in \mathcal{G}_{n}(d)$ with $\alpha(G)=n-d$ has the form $G=H+I_{n-d}$ for a graph $H$ of order $d$ and the empty graph $I_{n-d}$ on $n-d$ vertices. (Recall that for graphs $G, G^{\prime}$ with disjoint vertex sets, the sum $G+G^{\prime}$ denotes the graph obtained by adding all edges between them.) Let $K_{a, b}^{*}$ denote the graph $K_{a}+I_{b}$.

Denote by $X(G)$ the size of an independent set chosen uniformly at random from $\mathcal{I}(G)$. Recall that $X$ is stochastically dominated by $Y$, denoted by $X \leq_{s} Y$, if $\mathbb{P}(X \leq t) \geq \mathbb{P}(Y \leq t)$ for each $t$.

If $G \in \mathcal{G}_{n}(d)$ satisfies $\alpha(G)=n-d$ and $G$ is not $K_{d, n-d}^{*}$, then $G$ is (isomorphic to) a proper subgraph of $K_{d, n-d}^{*}$, and so $i(G)>i\left(K_{d, n-d}^{*}\right)$; and it follows that $\mathbb{P}(X(G) \leq t)<\mathbb{P}\left(X\left(K_{d, n-d}^{*}\right) \leq t\right)$ for $t=0$ and $t=1$. Hence it is not the case that $X(G) \leq_{s} X\left(K_{d, n-d}^{*}\right)$. Nevertheless, our second theorem shows that, if we ignore independent sets of size at most 1 , then of all graphs in $\mathcal{G}_{n}(d)$, the graph $K_{d, n-d}^{*}$ is the unique graph yielding the largest random independent sets.

Theorem 2. Let $1 \leq d=d(n)=o\left(n^{1 / 3}\right)$. Then for all sufficiently large $n$, for each graph $G \in \mathcal{G}_{n}(d)$ other than $K_{d, n-d}^{*}$, we have

$$
\mathbb{P}(X(G) \geq t)<\mathbb{P}\left(X\left(K_{d, n-d}^{*}\right) \geq t\right) \quad \text { for each } t=3, \ldots, n-d,
$$

and if $\alpha(G)<n-d$ then this inequality holds also for $t=1$ and 2 .

This yields directly:

Corollary 3. If $d$ is as above, then for all sufficiently large $n$, for each graph $G \in \mathcal{G}_{n}(d)$

$$
X(G) \leq_{s} \max \left\{2, X\left(K_{d, n-d}^{*}\right)\right\},
$$

and

$$
\text { if } \alpha(G)<n-d \text { then } X(G) \leq_{s} X\left(K_{d, n-d}^{*}\right) \text {. }
$$


Also, since $\mathbb{E}(X)=\sum_{t \geq 1} \mathbb{P}(X \geq t)$, we may obtain almost directly:

Corollary 4. If $1 \leq d=d(n)=o\left(n^{1 / 3}\right)$, then for all sufficiently large $n$, for each graph $G \in \mathcal{G}_{n}(d)$ other than $K_{d, n-d}^{*}$, we have

$$
\mathbb{E}(X(G))<\mathbb{E}\left(X\left(K_{d, n-d}^{*}\right)\right)<(n-d) / 2 .
$$

In order to prove these results, it turns out that the 'growth rates' $\alpha_{k}$ of the numbers of independent sets are crucial quantities. For a graph $G$ and positive integer $k \leq \alpha(G)$, let $\alpha_{k}(G):=\frac{i_{k}(G)}{i_{k-1}(G)}$. Thus $\alpha_{k}(G)$ is $1 / k$ times the average number of extensions of an independent $(k-1)$-set to an independent $k$-set in $G$; or (roughly) the 'average number of extensions per vertex' at size $k$.

To prove Theorem 1 we use two lemmas, one on growth rates $\alpha_{k}(G)$ and one on the 'base case' $i_{3}(G)$. To prove Theorem 2 we need one further lemma, a general result on growth rates and stochastic domination.

We adopt the following notations. For a graph $G$ and integer $d$ let $A=A(G, d)=\{v \in V(G): \operatorname{deg}(v)>d\}$ and $B=V(G) \backslash A$; and let $a=|A|$, $b=|B|$. Also recall the standard notation that, if $U$ is a set of vertices in $G$, then the neighbourhood $\Gamma(U)$ is the set of neighbours of vertices in $U$, and the closed neighbourhood $\Gamma[U]$ is $\Gamma(U) \cup U$.

Lemma 5. (a) For each $1 \leq d<n$ and $G \in \mathcal{G}_{n}(d)$, we have $\alpha_{k}(G) \leq$ $\alpha_{k}\left(K_{d, n-d}^{*}\right)$ for each $3 \leq k \leq \alpha(G)$.

(b) Let $1 \leq d=d(n)=o\left(n^{1 / 3}\right)$. Then for all sufficiently large $n$, for each $G, K \in \mathcal{G}_{n}(d)$ with $\alpha(G)<n-d=\alpha(K)$, we have $\alpha_{k}(G)<\alpha_{k}(K)$ for each $4 \leq k \leq \alpha(G)$.

Proof. Let $3 \leq k \leq \alpha(G)$. Since each vertex degree in $G$ is at least $d$, each $I \in \mathcal{I}_{k-1}(G)$ can be extended to at most $n-d-k+1$ independent $k$-sets. Call $I$ good if this upper bound is attained, and otherwise call $I$ bad. Note that $I$ is good if and only if $|\Gamma(I)|=d$, if and only if each vertex in $I$ has the same set of $d$ neighbours. Also, each $I$ is good if $G$ is $K_{d, n-d}^{*}$.

Since each independent $k$-set contains exactly $k$ independent $(k-1)$-sets, we have $i_{k-1}(G)(n-d-k+1) \geq k i_{k}(G)$. Hence, $\alpha_{k}(G) \leq \frac{n-d-k+1}{k}$. But $\alpha_{k}\left(K_{d, n-d}^{*}\right)=\frac{n-d-k+1}{k}$ for $k=3, \ldots, n-d$. This establishes part (a).

Now we prove part (b). Let $4 \leq k \leq \alpha(G)$. Suppose first that $k \geq d+2$. Let $J$ be an independent set in $G$ of size $\alpha(G) \leq n-d-1$. Let $W$ be a set of $d+1$ vertices outside $J$, and note that each vertex in $W$ has at least one neighbour in $J$. Since $k-1 \geq d+1$ we may pick a $(k-1)$ subset $I$ of $J$ with $\Gamma(I) \supseteq W$, and so $I$ is bad. Now, since there is a bad 
independent $(k-1)$-set, $\alpha_{k}(G)<\frac{n-d-k+1}{k}$. Further, $\alpha_{k}(K)=\frac{n-d-k+1}{k}$ for each $k=d+2, \ldots, n-d$, so this case is done; and so to prove part (b) we may assume that $4 \leq k \leq d+1$.

Assume also that $n>2 d$ (as we may). Write $K=H+I_{n-d}$ for some graph $H$ of order $d$. Then $\alpha_{k}(K)=\frac{\left(\begin{array}{c}n-d \\ k\end{array}\right)+i_{k}(H)}{\left(\begin{array}{c}n-d \\ k-1\end{array}\right)+i_{k-1}(H)}$. Since $i_{k-1}(H) \leq\left(\begin{array}{c}d \\ k-1\end{array}\right)$, for each $k \leq d+1$,

$$
\alpha_{k}(K) \geq \frac{\left(\begin{array}{c}
n-d \\
k
\end{array}\right)}{\left(\begin{array}{c}
n-d \\
k-1
\end{array}\right)+\left(\begin{array}{c}
d \\
k-1
\end{array}\right)}>\frac{n-d-k+1}{k}\left(1-\frac{\left(\begin{array}{c}
d \\
k-1
\end{array}\right)}{\left(\begin{array}{l}
n-d \\
k-1
\end{array}\right)}\right) .
$$

Let $p$ and $q$ denote the numbers of good and bad sets in $\mathcal{I}_{k-1}(G)$ respectively, so $p+q=i_{k-1}(G)$. Then

$$
k i_{k}(G) \leq p(n-d-k+1)+q(n-d-k)=(p+q)(n-d-k+1)-q,
$$

so

$$
\alpha_{k}(G) \leq \frac{n-d-k+1}{k}-\frac{q}{k(p+q)} .
$$

Assume for a contradiction that $\alpha_{k}(G) \geq \alpha_{k}(K)$. Then it follows using (3) and (4) that

$$
\frac{q}{p+q} \leq(n-d-k+1)\left(\begin{array}{c}
d \\
k-1
\end{array}\right) /\left(\begin{array}{l}
n-d \\
k-1
\end{array}\right)<\frac{d^{k-1}}{(n-d-k+1)^{k-2}} .
$$

Observe that, since $k \geq 4$, the final bound above is $O\left(d^{3} n^{-2}\right)=o\left(n^{-1}\right)$. Thus certainly $p>0$.

Claim: For each good independent $(k-1)$-set $I$ in $G$ there is a vertex $w \notin I \cup \Gamma(I)$ such that $\Gamma(w) \neq \Gamma(I)$.

We will prove the claim later: suppose for now that it holds. Then from each good independent $(k-1)$-set $I$ we may construct a bad independent $(k-1)$-set $I^{\prime}$ by deleting a vertex $u$ from $I$ and adding a vertex $w$ as in the claim. This gives at least $p(k-1) \geq 3 p$ constructions. Also, in each bad independent $(k-1)$-set $I^{\prime}$ which has been constructed, we can identify the vertex $w$ added (since the other $k-2 \geq 2$ vertices all have the same neighbourhood). Thus each bad independent $(k-1)$-set $I^{\prime}$ is constructed at most $n-k+1 \leq n-3$ times. Hence

$$
q \geq 3 p /(n-3)>p /(n-1)
$$

and so $q /(p+q)>1 / n$, which contradicts (5) (for $n$ sufficiently large, since $k \geq 4)$. 
It remains to prove the claim. Recall that $B=\{v \in V(G): \operatorname{deg}(v)=d\}$. Let $I$ be a good independent $(k-1)$-set. Note that $I \subseteq B$ and $|\Gamma(I)|=d$. If $|A|=a \geq d+1$ then for $w$ we may pick any vertex in $A \backslash \Gamma(I)$. So we may assume that $a \leq d$.

Let $B_{1}=\{v \in B: \Gamma(v) \cap B \neq \emptyset\}$ and $B_{2}=B \backslash B_{1}$. Since $\alpha(G)<n-d \leq$ $|B|$ we have $E(B) \neq \emptyset$ and so $B_{1} \neq \emptyset$. Either $I \subseteq B_{1}$ or $I \subseteq B_{2}$, since each vertex in $I$ has the same set of $d$ neighbours. If $I \subseteq B_{1}$ then $I \subseteq \Gamma(v)$ for some $v \in B_{1}$, and so for $w$ we may pick any vertex not in $\Gamma(I) \cup \Gamma(v)$ (at least $n-2 d \geq 1$ choices). If $I \subseteq B_{2}$ then for $w$ we may pick any vertex in $B_{1}$. This completes the proof of the claim, and we are done.

The previous lemma concerns ratios; the next considers the base case. Of graphs in $\mathcal{G}_{n}(d)$, clearly a $d$-regular graph has the most independent 2-sets: we look at the number $i_{3}$ of independent 3-sets. We first give a formula for $i_{3}(G)$ for any graph $G$. Let $t_{i}$ be the number of induced subgraphs of $G$ on three vertices with $i$ edges. Then

$$
\begin{aligned}
\left(\begin{array}{l}
n \\
3
\end{array}\right) & =t_{0}+t_{1}+t_{2}+t_{3}, \\
e(G)(n-2) & =t_{1}+2 t_{2}+3 t_{3}, \\
\sum_{v_{i} \in V(G)}\left(\begin{array}{c}
\operatorname{deg}\left(v_{i}\right) \\
2
\end{array}\right) & =t_{2}+3 t_{3} .
\end{aligned}
$$

Hence,

$$
i_{3}(G)=\left(\begin{array}{l}
n \\
3
\end{array}\right)-e(G)(n-2)+\sum_{v_{i} \in V(G)}\left(\begin{array}{c}
\operatorname{deg}\left(v_{i}\right) \\
2
\end{array}\right)-t(G),
$$

where $t(G)=t_{3}$ is the number of triangles. For example, if $G$ is a $d$-regular graph then

$$
\begin{aligned}
i_{3}(G) & =\left(\begin{array}{l}
n \\
3
\end{array}\right)-\frac{1}{2} d n(n-2)+n\left(\begin{array}{l}
d \\
2
\end{array}\right)-t(G) \\
& =\left(\begin{array}{c}
n-d \\
3
\end{array}\right)-\frac{1}{2} d n+\frac{1}{6} d\left(d^{2}+3 d+2\right)-t(G) .
\end{aligned}
$$

Lemma 6. Let $1 \leq d=d(n)=o\left(n^{1 / 3}\right)$. For all sufficiently large $n$, if $G, K \in \mathcal{G}_{n}(d)$ are such that $\alpha(G)<n-d=\alpha(K)$, then $i_{3}(G) \leq i_{3}(K)-$ $n / 2+1$. 
Proof. Our proof relies on (6). Consider $G \in \mathcal{G}_{n}(d)$ with $\alpha(G)<n-d$. We first show that we may assume without loss of generality that the set $A$ of vertices of degree $>d$ is a non-empty independent set, and then that it suffices to prove (7) below; then we prove (7) by considering four cases for $a=|A|$.

Suppose that $G$ is $d$-regular. Then by the above we have

$$
i_{3}(G) \leq\left(\begin{array}{c}
n-d \\
3
\end{array}\right)-\frac{1}{2} d n+\frac{1}{6} d\left(d^{2}+3 d+2\right) .
$$

But $i_{3}(K) \geq\left(\begin{array}{c}n-d \\ 3\end{array}\right)$. Thus, if $d=1$ then

$$
i_{3}(G) \leq\left(\begin{array}{c}
n-d \\
3
\end{array}\right)-n / 2+1 \leq i_{3}(K)-n / 2+1
$$

and if $d \geq 2$ then

$$
i_{3}(G) \leq\left(\begin{array}{c}
n-d \\
3
\end{array}\right)-n+O\left(d^{3}\right) \leq i_{3}(K)-n / 2
$$

for $n$ sufficiently large. Hence we may assume that $G$ is not regular, and so $A$ is non-empty.

Now repeatedly delete edges between vertices of degree $>d$, as long as $G$ keeps satisfying $\alpha(G)<n-d$. We end up with some graph $G^{\prime} \in \mathcal{G}_{n}(d)$ with $\alpha\left(G^{\prime}\right)<n-d$. Suppose that there is an edge $u v \in E^{\prime}\left(A^{\prime}\right)$ after this step (we use $E^{\prime}$ and $A^{\prime}$ to refer to $\left.G^{\prime}\right)$. Then there exists an $(n-d)$-set $I$ such that $E^{\prime}(I)=\{u v\}$. Let $J=V\left(G^{\prime}\right) \backslash I$, so $|J|=d$. Since $\operatorname{deg}_{G^{\prime}}(u), \operatorname{deg}_{G^{\prime}}(v)>d$ and $\operatorname{deg}_{G^{\prime}}(w) \geq d$ for each other vertex $w \in I$, every possible edge between $I$ and $J$ is present in $G^{\prime}$. Therefore, since there are $(n-d-2) 3$-subsets of $I$ containing $u$ and $v$,

$$
i_{3}(G) \leq i_{3}\left(G^{\prime}\right) \leq\left(\begin{array}{c}
n-d \\
3
\end{array}\right)-(n-d-2)+\left(\begin{array}{l}
d \\
3
\end{array}\right)<\left(\begin{array}{c}
n-d \\
3
\end{array}\right)-\frac{n}{2}
$$

for large $n$, since $d=o\left(n^{1 / 3}\right)$. Hence, we may assume that $A$ is independent.

For each $v_{i} \in A$, let $r_{i}=\operatorname{deg}\left(v_{i}\right)$. Observe that $2 e(G)=\sum_{i} r_{i}+(n-a) d$. 
Thus, from (6),

$$
\begin{aligned}
& 2 i_{3}(G)-2\left(\begin{array}{l}
n \\
3
\end{array}\right) \\
= & -\left[\sum_{i=1}^{a} r_{i}+(n-a) d\right](n-2)+\sum_{i=1}^{a} r_{i}\left(r_{i}-1\right)+(n-a) d(d-1)-2 t(G) \\
= & \sum_{i=1}^{a} r_{i}\left(r_{i}-n+1\right)-(n-a) d(n-d-1)-2 t(G) \\
= & -d n(n-d-1)+h_{d}(G),
\end{aligned}
$$

where

$$
h_{d}(G)=\sum_{i=1}^{a} r_{i}\left(r_{i}-n+1\right)+a d(n-1-d)-2 t(G) .
$$

Thus

$$
\begin{aligned}
i_{3}(G) & =\left(\begin{array}{l}
n \\
3
\end{array}\right)-\frac{1}{2} d n(n-d-1)+\frac{1}{2} h_{d}(G) \\
& =\left(\begin{array}{c}
n-d \\
3
\end{array}\right)-\frac{1}{2} d n+\frac{1}{6} d\left(d^{2}+3 d+2\right)+\frac{1}{2} h_{d}(G) .
\end{aligned}
$$

Observe that here only $\frac{1}{2} h_{d}(G)$ varies with $G \in \mathcal{G}_{n}(d)$. Since $i_{3}(K) \geq\left(\begin{array}{c}n-d \\ 3\end{array}\right)$, by the last equality

$$
h_{d}(K) \geq d n-\frac{1}{3} d\left(d^{2}+3 d+2\right)=(1+o(1)) d n .
$$

Thus it suffices to show that

$$
h_{d}(G) \leq(d-1) n+O\left(d^{2}\right)
$$

and the remainder of the proof is devoted to establishing this result.

Recall that we are assuming that in $G$ the set $A$ of vertices of degree $>d$ is independent. Thus $d+1 \leq r_{i} \leq n-a$ for each $i=1, \ldots, a$. Consider the function $g(x)=x(x-n+1)=-x(n-1-x)$ for real $x$. This is decreasing for $x<(n-1) / 2$ and increasing for $x>(n-1) / 2$. We now break the proof of (7) into four cases: $a \geq d+2, a=d+1, a=d$, and $1 \leq a \leq d-1$.

Suppose that $a \geq d+2$. Then each $d+1 \leq r_{i} \leq n-d-2$, so $g\left(r_{i}\right) \leq$ $(d+1)(d+2-n)$. Hence,

$$
\begin{aligned}
h_{d}(G) & \leq a(d+1)(d+2-n)+a d(n-1-d) \\
& =a(-n+2 d+2)
\end{aligned}
$$


and so (7) holds.

Suppose that $a=d+1$. Then $d+1 \leq r_{i} \leq n-d-1$ for each $i$, and $\sum_{i=1}^{a} r_{i} \leq d(n-a)=d(n-d-1)$. Thus at most $d-1$ of the $r_{i}$ are equal to $n-d-1$, and so

$\sum_{i=1}^{a} g\left(r_{i}\right) \leq-(d-1) d(n-d-1)-2(d+1)(n-d-2)=-n\left(d^{2}+d+2\right)+O\left(d^{3}\right)$.

Hence $h_{d}(G) \leq-2 n+O\left(d^{3}\right)$, and so (7) holds.

Suppose that $a=d$. Since $\alpha(G)<n-d, e(B)>0$. It follows that $\sum_{i=1}^{a} r_{i} \leq d(n-d)-2 \leq d(n-d)-1$. Hence not all $d$ of the $r_{i}$ are equal to $n-d$, and so

$$
\begin{aligned}
h_{d}(G) & \leq(d-1)(n-d)(1-d)+(n-d-1)(-d)+d^{2}(n-1-d) \\
& =(d-1) n+O\left(d^{2}\right)
\end{aligned}
$$

as required.

Finally, suppose that $1 \leq a \leq d-1$. Consider $v_{i} \in A$. Suppose that $r_{i} \geq n-d-1$. Then the edge-boundary of $\Gamma\left(v_{i}\right)$ has size at most $r_{i} a+(n-$ $\left.a-r_{i}\right) d \leq r_{i} a+d(d+1-a)$, and so $2 e\left(\Gamma\left(v_{i}\right)\right) \geq r_{i}(d-a)-d(d+1-a)$. Hence, twice the number of triangles containing $v_{i}$ is at least $r_{i}(d-a)-d(d+1-a)$. Also, using first that $r_{i} \leq n-a$ and then that $r_{i} \geq n-d-1$ we have

$$
g\left(r_{i}\right)-r_{i}(d-a)=r_{i}\left(r_{i}-n+1-d+a\right) \leq r_{i}(1-d) \leq(n-d-1)(1-d) .
$$

On the other hand, if $r_{i} \leq n-d-2$, then $g\left(r_{i}\right) \leq(d+1)(d-n+2)$. Let $l=\left|\left\{i: r_{i} \geq n-d-1\right\}\right|$. Then $h_{d}(G)$ is at most

$$
\begin{aligned}
& \sum_{i: r_{i} \geq n-d-1}\left[g\left(r_{i}\right)-r_{i}(d-a)+d(d+1-a)\right]+\sum_{i: r_{i} \leq n-d-2} g\left(r_{i}\right)+a d(n-1-d) \\
\leq & l[(n-d-1)(1-d)+d(d+1-a)]+(a-l)(d+1)(d-n+2)+a d(n-1-d) \\
\leq & a n+O\left(d^{2}\right) \leq(d-1) n+O\left(d^{2}\right)
\end{aligned}
$$

as required.

With the last two lemmas, we may now prove Theorem 1, establishing a stronger version of the conjecture of Galvin [3] mentioned earlier.

Proof of Theorem 1. If $\alpha(G)=n-d$ then $G$ is (isomorphic to) a supergraph of $K_{d, n-d}$ and the result is trivial: so we may assume that $\alpha(G)<n-d$. Let us also assume that $n$ is large. Let $K \in \mathcal{G}_{n}(d)$ with $\alpha(K)=n-d$. 
Since $i_{3}(G) \leq i_{3}(K)-n / 2+1$ by Lemma $6(\mathrm{~b})$, by Lemma 5 we have $i_{k}(G)<i_{k}(K)$ for all $k \geq 3$. In fact, $i_{4}(G)<i_{4}(K)-\Omega\left(n^{2}\right)$ since $\alpha_{4}(K)=$ $\Omega(n)$. On the other hand, $e(G) \geq d n / 2$, so that $i_{2}(G)-i_{2}(K) \leq d n / 2$. Thus $i_{2}(G)+i_{4}(G)<i_{2}(K)+i_{4}(K)$, and we are done.

To prove Theorem 2, as well as the two corollaries, we need one further lemma, which is a general result on growth rates and stochastic domination, adapted from Lemma 2.4 of [9]. Given a finite sequence of positive real numbers $x=\left(x_{0}, x_{1}, \ldots, x_{s}\right)$, let $S(x)=\sum_{k \geq 0} x_{k}$. Define a random variable $X=X(x)$ by $\mathbb{P}(X=k)=x_{k} / S(x)$.

Lemma 7. Let $x_{0}, y_{0}>0$, let $1 \leq a \leq b$ be integers, and let $\alpha_{1}, \ldots, \alpha_{a}>0$ and $\beta_{1}, \ldots, \beta_{b}>0$. For $i=1, \ldots$, a, let $x_{i}=x_{0} \prod_{0<j<i} \alpha_{j}$; and for $i=1, \ldots, b$, let $y_{i}=y_{0} \prod_{0<j<i} \beta_{j}$. Let $x=\left(x_{0}, x_{1}, \ldots, x_{a}\right)$ and $y=$ $\left(y_{0}, y_{1}, \ldots, y_{b}\right)$, and denote $X(x)$ by $X$ and $X(y)$ by $Y$. If $\alpha_{i} \leq \beta_{i}$ for each $i=1, \ldots, a$, then $X \leq_{s} Y$. Further, if these conditions hold, and $\left(\alpha_{1}, \ldots, \alpha_{a}\right) \neq\left(\beta_{1}, \ldots, \beta_{b}\right)$, then

$$
\mathbb{P}(X \geq t)<\mathbb{P}(Y \geq t) \text { for each } t=1, \ldots, b .
$$

Proof. By replacing $y_{a}$ by $\sum_{j>a} y_{j}$, we may assume that $b=a$. It suffices to consider the case when $\alpha_{i}=\beta_{i}$ for all $i$ except $j_{0}$ where $\alpha_{j_{0}}<\beta_{j_{0}}$. Since $\mathbb{P}(X \leq a)=\mathbb{P}(Y \leq a)=1$, it suffices to prove $\mathbb{P}(X \leq t)>\mathbb{P}(Y \leq t)$ for $t=0, \ldots, a-1$. Note that we may rescale $x_{i}, y_{i}$ 's without changing the distribution.

Suppose $t$ satisfies $0 \leq t \leq j_{0}-1$. Rescale to $x_{0}=y_{0}=1$. Then $x_{i}=y_{i}$ for all $i \leq t$ and $S(x)<S(y)$. So $\mathbb{P}(X \leq t)=\frac{\sum_{i \leq t} x_{i}}{S(x)}>\frac{\sum_{i \leq t} y_{i}}{S(y)}=\mathbb{P}(Y \leq t)$.

For $t$ such that $j_{0} \leq t \leq a-1$, we rescale to $x_{j_{0}}=y_{j_{0}}$. Then $x_{i}=y_{i}$ for all $i=j_{0}, j_{0}+1, \ldots, a$ and $S(x)>S(y)$. Hence, $\mathbb{P}(X>t)<\mathbb{P}(Y>t)$ and so $\mathbb{P}(X \leq t)>\mathbb{P}(Y \leq t)$.

Proof of Theorem 2. There are two cases, depending on whether $\alpha(G)<$ $n-d$ or $\alpha(G)=n-d$.

(a) Let $G \in \mathcal{G}_{n}(d)$ with $\alpha(G)<n-d$. For $k \geq 1$, let $\alpha_{k}^{*}$ denote $\alpha_{k}\left(K_{d, n-d}^{*}\right)$. Then $\alpha_{1}(G)=\alpha_{1}^{*}=n$. By Lemma $5(\mathrm{a}), \alpha_{k}(G) \leq \alpha_{k}^{*}$ for $3 \leq k \leq \alpha(G)$.

If $\alpha_{2}(G) \leq \alpha_{2}^{*}$ then directly from Lemma 7 we have $\mathbb{P}(X(G) \geq t)<$ $\mathbb{P}\left(X\left(K_{d, n-d}^{*}\right) \geq t\right)$ for each $t=1, \ldots, n-d$, and we are done. So we may suppose that $\alpha_{2}(G)>\alpha_{2}^{*}$; that is $i_{2}(G)>i_{2}^{*}$, where $i_{k}^{*}$ denotes $i_{k}\left(K_{d, n-d}^{*}\right)$. 
Let $x$ be the $i_{k}$-vector for $G$ (up to $x_{n-d}$ ), let $z$ be the $i_{k}$-vector for $K_{d, n-d}^{*}$, and let $y$ agree with $x$ in the first three places, and agree with $z$ in the remaining places; that is,

$$
\begin{gathered}
x=\left(x_{0}, x_{1}, \ldots, x_{n-d}\right)=\left(1, n, i_{2}(G), i_{3}(G), i_{4}(G), \ldots, i_{n-d}(G)\right), \\
y=\left(y_{0}, y_{1}, \ldots, y_{n-d}\right)=\left(1, n, i_{2}(G), i_{3}^{*}, i_{4}^{*}, \ldots, i_{n-d}^{*}\right)
\end{gathered}
$$

and

$$
z=\left(z_{0}, z_{1}, \ldots, z_{n-d}\right)=\left(1, n, i_{2}^{*}, i_{3}^{*}, i_{4}^{*}, \ldots, i_{n-d}^{*}\right) .
$$

Let $3 \leq t \leq n-d$. By Lemma 5 (b) with $K=K_{d, n-d}^{*}$, for each $4 \leq k \leq \alpha(G)$ we have $\frac{x_{k}}{x_{k-1}} \leq \frac{y_{k}}{y_{k-1}}$. Moreover, by Lemma $6, i_{3}(G)<i_{3}^{*}$ so that $\frac{x_{3}}{x_{2}}<\frac{y_{3}}{y_{2}}$. Then by Lemma 7 ,

$$
\mathbb{P}(X(G) \geq t)=\mathbb{P}(X(x) \geq t)<\mathbb{P}(X(y) \geq t) .
$$

Also

$$
\mathbb{P}(X(y) \geq t)<\mathbb{P}(X(z) \geq t)=\mathbb{P}\left(X\left(K_{d, n-d}^{*}\right) \geq t\right)
$$

since $S(y)<S(z)$. Hence $\mathbb{P}(X(G) \geq t)<\mathbb{P}\left(X\left(K_{d, n-d}^{*}\right) \geq t\right)$ as required.

To complete the proof for this case, note that by Theorem $1, i(G)<$ $i\left(K_{d, n-d}^{*}\right)$, so that

$$
\mathbb{P}(X(G) \leq 0)=1 / i(G)>1 / i\left(K_{d, n-d}^{*}\right)=\mathbb{P}\left(X\left(K_{d, n-d}^{*}\right) \leq 0\right),
$$

and similarly

$$
\mathbb{P}(X(G) \leq 1)=(1+n) / i(G)>(1+n) / i\left(K_{d, n-d}^{*}\right)=\mathbb{P}\left(X\left(K_{d, n-d}^{*}\right) \leq 1\right) .
$$

(b) It remains to consider the case when $\alpha(G)=n-d$ and $G$ is not $K_{d, n-d}^{*}$. Then $G$ may be obtained from $K_{d, n-d}^{*}$ by deleting at least one edge from the $K_{d}$ part. Thus $i(G)>i\left(K_{d, n-d}^{*}\right)$; and the $i_{k}$-vector $x$ of $G$ may be obtained from the $i_{k}$-vector $z$ for $K_{d, n-d}^{*}$ by adding positive integers to some entries amongst the first $d+1$ including adding at least 1 to $z_{2}$. It is immediate that $\mathbb{P}(X(x) \geq t)<\mathbb{P}(X(z) \geq t)$ for each $t=d+1, \ldots, n-d$. Let $2 \leq t \leq d-1$. Then

$$
\mathbb{P}(X(z) \leq t)=\frac{\sum_{i=0}^{t} z_{i}}{S(z)}
$$

To obtain $\mathbb{P}(X(x) \leq t)$ from the last ratio we add at least 1 to the numerator and at most $2^{d}$ to the denominator. Thus the numerator increases by a factor $\left(1+\Omega\left(n^{-d}\right)\right)$ and the denominator increases by a factor at most $\left(1+2^{-(n-2 d)}\right)$. So overall the ratio increases (for large $n$ ), that is $\mathbb{P}(X(z) \leq t)<\mathbb{P}(X(x) \leq$ $t$ ), as required. 
We noted earlier that Corollary 3 follows directly from Theorem 2, so it remains only to prove Corollary 4.

Proof of Corollary 4. If $\alpha(G)<n-d$, the result follows directly from (2). Suppose then that $\alpha(G)=n-d$, and let $n$ be sufficiently large that $\mathbb{E}\left[X\left(K_{d, n-d}^{*}\right)\right] \geq d$. Then the average size of the sets which are independent in $G$ but not in $K_{d, n-d}^{*}$ is at most $d \leq \mathbb{E}\left[X\left(K_{d, n-d}^{*}\right)\right]$, and so $\mathbb{E}[X(G)] \leq \mathbb{E}\left[X\left(K_{d, n-d}^{*}\right)\right]$.

We remark that with an analogous method, a weighted version of the statements can be proved. Let $I(G, \lambda)=\sum_{k>0} i_{k}(G) \lambda^{k}$ be the independent set polynomial of $G$ ([4], [8]). Instead of a uniform sampling of independent sets of $\mathcal{I}(G)$, we fix $\lambda>0$ and pick a given independent $k$-set with probability $\lambda^{k} / I(G, \lambda)$. Then under this sampling, the analogous versions of Theorem 2 and its corollaries hold.

\section{References}

[1] T. Carroll, D. Galvin and P. Tetali, Matchings and independent sets of a fixed size in regular graphs, J. Combin. Theory Ser. A 116 (2009), $1219-1227$.

[2] J. Cutler and A.J. Radcliffe, Extremal problems for independent set enumeration Electron. J. Combin. 18 (2011), \# P169.

[3] D. Galvin, Two problems on independent sets in graphs, Discrete Math. 311 (2011), 2105-2112.

[4] I. Gutman and F. Harary, Generalizations of the matching polynomial, Utilitas Mathematica 24 (1983) 97-106.

[5] J. Kahn, An entropy approach to the hard-core model on bipartite graphs, Combin. Probab. Comput. 10 (2001), 219-237.

[6] S.B. Lin and C. Lin, Trees and forests with large and small independent indices, Chinese J. Math. 23 (1995), 199-210.

[7] H. Prodinger and R.F. Tichy, Fibonacci numbers of graphs, Fibonacci Quart. 20 (1982), 16-21.

[8] A.D. Scott and A.D. Sokal, The repulsive lattice gas, the independentset polynomial, and the Lovász local lemma, J. Stat. Phys. 118 (2005), $1151-1261$. 
[9] C. McDiarmid, A. Steger and D. Welsh, Random planar graphs. J. Combin. Theory B 93 (2005) 187-205.

[10] Y. Zhao, The number of independent sets in a regular graph, Combin. Probab. Comput. 19 (2010), 315-320. 